\title{
Developing New Molecules for the Treatment of Painful Diabetic Peripheral Neuropathy: Is it Feasible given the Magnitude of the Placebo Response in Proof of Concept Clinical Studies?
}

\author{
Domenico Merante $^{1 *}$, George Skouteris ${ }^{1}$ and Rayaz Malik ${ }^{2}$ \\ ${ }^{1}$ Daiichi Sankyo Development Ltd., UK \\ ${ }^{2}$ Centre of Endocrinology and Diabetes - Institute of Human Development, University of Manchester,UK
}

\begin{abstract}
Randomised, double blind, placebo controlled studies are necessary in the neuropathic pain clinical research arena. Few drugs are approved for the indication of painful Diabetic Peripheral Neuropathy (DPN): typically these drugs lose their therapeutic response over time and have a far from ideal tolerability risk profile. In addition to that, the response to placebo continues to represent a significant challenge in clinical trials of painful DPN, as well as other neuropathic pain condition. Hence to date few molecules have progressed to phase 3 in painful DPN and even fewer have gained approval. Thus many studies failed because the primary endpoint of statistical superiority to placebo and a clinically relevant reduction of ADPS (Average Daily Pain Score) were not achieved. Any attempt to mitigate the placebo response in this condition may allow future new drugs to successfully progress from Proof of Concept to phase 3 of clinical development and hopefully to their approval. As of today this remains a relevant challenge in a therapeutic area of a well-recognized unmet medical need. The dangers of treating acute and chronic pain with opioids, Non-Steroidal Anti-Inflammatory Drugs (NSAIDs) or acetaminophen are considerable. By mitigating the placebo response in neuropathic pain clinical trials, this may expedite the discovery, selection and development of improved analgesic treatments for the benefit of public health. In addition, a better understanding of subjects' typology would be of great help to identify those who really need a more specific pharmacological treatment approach from those subjects who don't and might benefit instead from alternative non-pharmacological approaches.
\end{abstract}

\section{Magnitude of the Placebo Response in Painful Diabetic Peripheral Neuropathy}

The pathogenesis of pain in diabetic peripheral neuropathy (painful DPN) involves both peripheral and central mechanisms [1] The insult of diabetes, in fact, impacts the nervous system from the periphery to the brain. Peripheral mechanisms are due to changes in calcium channel distribution and expression; changes in sodium channel distribution and expression; altered neuropeptide expression; sympathetic sprouting; peripheral sensitization; loss of spinal inhibitory control; altered peripheral blood flow; axonal atrophy, degeneration or regeneration; damage to small fibers; increased glycaemic flux. Central mechanisms include central sensitization; changes in the balance of facilitation/inhibition within descending pathways; increased thalamic vascularity $[1,2]$. In clinical studies conducted in painful DPN the primary efficacy assessment is typically based upon the 11 point Numerical Rating Pain Scale (NRS) with $0=$ no pain and $10=$ worst possible pain. Pain can also be measured by the VAS (Visual Analogue Scale), which is a unidimensional validated measure of pain intensity and has been widely used in diverse adult populations, including those with painful DPN. As the NRS scale, for pain intensity, the VAS scale is most commonly anchored by "no pain" (score of 0 ) and "pain as bad as it could be" or "worst imaginable pain" (score of 100 [100-mm scale]). The pain is assessed by the patients in their daily pain score through paper or electronic diaries. The patients score their pain during the last 24 hours at awakening. Typically the study primary endpoint is the weekly mean pain score at endpoint (=endpoint mean score) often defined as the mean pain score for the last $4 / 7$ available pain diary entries while on study medication. Responder rates are defined as $30 \%$ and $50 \%$ reduction in pain score as compared to baseline. Numerous secondary efficacy measurement scales can be used in painful DPN studies. These include the Short-Form McGill Pain Questionnaire (SF-MPQ), BPI (Brief Pain Inventory), a Sleep interference scale, the global impression of change by clinician (CGIC), the global impression of change by patient (PGIC), the SF-36 and/or EQ-5D Quality of life Questionnaires, a mood assessment (profile of mood states [POMS] and the Hospital anxiety and depression scale [HADS] and others.

The placebo response plays a pivotal role in clinical trials of painful DPN [3]. From an analysis of 70 studies (10,297 patients) conducted specifically in painful DPN, the average response in terms of weighted mean differences was 13.96 (95\% CI 11.93 to 15.99) on the Visual Analogue Scale (VAS), utilized as the primary endpoint of the ADPS (Average Daily Pain Score). Placebo accounted for $62 \%$ of the response and was significantly higher compared to a parallel analysis carried out on Fibromyalgia $(\mathrm{P}<0.001)$, a syndrome also associated with neuropathic pain. From this analysis the placebo response was not associated with age, sex, and race, but indeed with year of study initiation, severity of baseline pain and effect size in active drug groups in both diseases.

Thus, significant efficacy in patients receiving placebo may obscure

*Corresponding author: Domenico Merante, Senior Director Clinical Development, Daiichi Sankyo Development Ltd. Chiltern Place, Chalfont Park, Gerrards Cross, Buckinghamshire-SL9 OBG, UK, Tel: 0044 (0) 1753482802; Fax: 0044 (0) 1753899107; E-mail: nmerante@dsd-eu.com

Received September 20, 2013; Accepted October 29, 2013; Published November 05, 2013

Citation: Merante D, Skouteris G, Malik R (2013) Developing New Molecules for the Treatment of Painful Diabetic Peripheral Neuropathy: Is it Feasible given the Magnitude of the Placebo Response in Proof of Concept Clinical Studies? J Diabetes Metab 4: 305. doi:10.4172/2155-6156.1000305

Copyright: (c) 2013 Merante D, et al. This is an open-access article distributed under the terms of the Creative Commons Attribution License, which permits unrestricted use, distribution, and reproduction in any medium, provided the original author and source are credited. 
Citation: Merante D, Skouteris G, Malik R (2013) Developing New Molecules for the Treatment of Painful Diabetic Peripheral Neuropathy: Is it Feasible given the Magnitude of the Placebo Response in Proof of Concept Clinical Studies? J Diabetes Metab 4: 305. doi:10.4172/21556156.1000305

Page 2 of 4

the potential analgesic effect of a promising new compound under development. The placebo effect can vary widely across analgesic trials and, in combination with regression to the mean and therapeutic misconception, can markedly decrease the effect size of a study. Regression to the mean refers to the observation that patients tend to enter clinical trials during a pain exacerbation and that pain intensity improves over the course of the study. Therapeutic misconception occurs when patients do not accurately understand the nature of a clinical trial and believe that they will receive an active drug. It is therefore important to instruct and educate patients entering a clinical trial by clearly explaining up front that they might receive an inactive placebo and that it is possible that the active drug is ineffective [4].

\section{Factors Associated with the Placebo Response Rate in Neuropathic Pain Studies}

The relationship between the placebo response rate and the other study characteristics was also investigated in another meta-analysis of 106 clinical trials characterised by mostly peripheral but also central neuropathic pain [5]. Differently from the analysis conducted by W. Häuser [3], the studies from this analysis in fact such as patients with neuropathic pain from conditions such as post-herpetic neuralgia, painful polyneuropathy, including painful DPN and other peripheral and central neuropathic pain-related conditions. The analysis showed that a greater placebo response was associated with greater medication response, longer trial duration, and the use of parallel group designs. Because outliers might have accounted for relationships between active medication and placebo response rates, the data was re-analysed and the Spearman rank order correlation coefficient between placebo and medication response was calculated. The repeated analysis still indicated the significant relationship between greater placebo response and greater medication response. There was a non-significant relationship between greater placebo response and larger sample size, but from this analysis the year of publication, baseline pain scoring, primary outcome measure, or pain condition were not significantly associated with placebo response. In addition, a linear multiple regression analysis was conducted to identify unique contributions of the study characteristics to the placebo response. The results of this analysis confirmed again that a greater placebo response was associated with a greater active medication response and use of parallel groups rather than cross-over designs.

The most evident difference from the analysis conducted by $\mathrm{W}$. Häuser [3] compared to the one by J. Katz [5] seems to be the positive correlation of the magnitude of the placebo response with the baseline pain score.

Also the correlation of the placebo response with year of study initiation and year of study publication diverged between the two analyses, even though the latter might not necessarily correlate with the first one as study results are not always published straight after their completion and, particularly in the past, negative results were quite likely not published at all. This risk has to be taken into account in the evaluation of both analyses and regarded as a limitation to full understanding of the data.

Also only the analysis from J. Katz [5] showed a positive correlation of the placebo response with parallel groups study designs and with trial duration. This is more difficult to explain. Due to the lack of 'intrasubject' comparative data, a parallel group study, differently from a cross-over design, might be more confounding to study subjects for their perception or belief to perceive any difference between an active drug and placebo. This might apply to their assessment of the pain score and to the tolerability profile of any new active medication, as well as to the placebo treatment and related response (placebo and nocebo effect).

As far as the study duration is concerned, there is today still controversy in terms of a positive correlation with placebo response. This is due to limited available evidence, which seems to indicate a positive correlation with the placebo response in painful DPN more likely to occur whilst subjects are in longer [6] rather than shorter-term studies [7].

All these differing factors between the outcomes from the two analyses certainly require further investigation in future research.

\section{Potential ways to Mitigate the Placebo Response in Painful DPN Studies \\ Including in the clinical study design since phase 2 Proof of Concept a placebo run-in period}

Proof-of-Concept (PoC) clinical trials are carefully designed to establish the safety of drug candidates in the target population and explore the relationship between the dose and desired activity, as either measured directly or by means of a surrogate.

Potential ways of mitigating the placebo response have been developed over the past few decades [8].

To date, the placebo response in painful DPN and in other pain conditions studies remains high and no definitive solution has been identified to mitigate this effect [9-11].

One of the most common reasons for screen failures is in fact the positive placebo response during the run-in period of the trial. Thus many molecules in clinical development have failed because the active tested medication was not found to be superior to placebo for the primary endpoint. Whilst testing a new molecule for painful DPN in the PoC phase, starting with a week, single blinded, placebo run-in period, instead of a selection of responders to an active drug, might be an unconventional but potentially favourable way to try to reduce the placebo effect. Patients who respond to placebo in this period of time (those with at least $30 \%$ decrease in pain VAS from baseline value at screening visit) should not be randomized into subsequent phases of the trial. These subjects definitely represent a subgroup of the placeboresponders, not all placebo responders, and as such can be filtered out through this run-in period before randomization. They will be excluded from the analysis of the efficacy study results.

\section{Minimizing staff and subject expectations}

Likewise in depressive disorders [12,13], studies in painful DPN primarily require the use of placebo in their study design in order to detect if the tested active medication is superior to placebo in pain scoring as the primary endpoint.

To further mitigate the placebo response, it may be useful to implement strategies to decrease overinflated patient and staff expectations of improvement. This can be achieved during training sessions at the sites and during study investigator meetings and training sessions with the study coordinator and monitoring meetings. It is important that subjects entering a clinical trial understand that neuropathic pain is a condition where a new active medication may not necessarily be superior to existing treatment and to placebo [14] Patients need to know the study design correctly and the possibility they may be on placebo rather than on active medications: this needs to be explained clearly, so false expectations can be avoided from study entry [15]. 
Citation: Merante D, Skouteris G, Malik R (2013) Developing New Molecules for the Treatment of Painful Diabetic Peripheral Neuropathy: Is it Feasible given the Magnitude of the Placebo Response in Proof of Concept Clinical Studies? J Diabetes Metab 4: 305. doi:10.4172/21556156.1000305

Page 3 of 4

In addition, any form of advertising, which has become nowadays quite common in clinical research in order to accelerate recruitment and enrolment timelines, should be discouraged. This in fact might increase subject's expectations and ultimately the placebo response, particularly in those subjects who are more prone to be psychologically influenced by such procedures.

\section{Identifying subjects more likely to respond to placebo}

Ultimately it may be appropriate to use novel approaches for identifying individuals more likely to respond to placebo. This approach should be utilized particularly in phase 2 Proof of Concept. We acknowledge that the attempt to identify subjects more likely to respond to placebo on its own might bias the results to responders and does not necessarily guarantee a successful phase 3 trial. Also the method is per se quite challenging, as to date there are no specifically identified and scientifically recognized patient phenotypes.

Future research is necessary to understand the clinical differences between clinically existing patient's typologies. This approach has been already utilized in neuropathic pain associated with fibromyalgia where subgroups of patients with different clinical profiles and degrees of depression/anxiety, with low or high or no control over pain, if low or very high catastrophizers, were identified [16]. This could similarly apply to painful DPN patients. As a start for more future research, recently two completely opposite pain phenotypes were hypothesized [17].

More investigation is needed to better understand the different clinical profiles in terms of placebo responders. Conversely, the understanding of these profiles could also have an impact on the identification of patients who could respond to available non pharmacological and pharmacological interventions.

Although it is possible that either pain drug naïve subjects with a shorter duration of painful DPN, either those with mood disorders, is more likely to be at higher risk to be placebo responders in clinical studies, as they are minimally exposed to approve neuropathic pain medications. Typically, pain drug-naïve subjects are more sensitive to a pain medication, but also to placebo. Over time they become less responsive to a pain drug and combination therapies maybe in fact often required. In addition, subjects affected by depression and mood disorders are more likely to be more psychologically 'influenced by a new study pain medication, but also by placebo $[18,19]$. Subjects may feel better during the clinical trial simply because of an improvement of their mood due to a close level of attention provided at the investigational site after their inclusion in the clinical trial. It is important to exclude in phase 2 PoC study those anti-depressant therapies, which can also exert positive effects on neuropathic pain (tricyclic anti-depressants and serotonin norepinephrine reuptake inhibitors), so any tested benefit from a new investigational pain medication will not be obscured and not related to any of those drugs often utilized as combination therapies in clinical practice. If a new pain medication receives regulatory approval, then additional clinical studies testing and comparing combination therapies with the approved drug should also be designed and conducted.

In addition and not less importantly, the potential identification of subjects more likely to respond to placebo needs to be part of the statistical analysis study plan, which should include the methodologies needed for the correct analyses of clinical results. In this regard, the missing data need to be handled by using the baseline observation carried forward (BOCF) instead of the last observation carried forward (LOCF) methodology, which often biases the analyses of such trials and makes the new investigational drug not statistically significant against placebo. Sensitivity analyses for different methods of handling missing data, including repeated mixed models, need to be conducted: this approach could help in meeting statistical criteria for efficacy of a newly promising investigational drug.

\section{Discussion}

In summary, the placebo response remains today a significant challenge in clinical trials of painful DPN and other neuropathic pain conditions. Hence to date, very few molecules have progressed to phase 3 in painful DPN and even fewer have gained approval. Thus several recent studies have failed because the primary endpoint of statistical superiority to placebo and a clinically relevant reduction of ADPS were not achieved $[6,20,21]$.

In trials of painful DPN the placebo response shows to increase over time and may often contribute to the lack of statistical significance in the primary end point [6]. High placebo responses are in fact not unusual in neuropathic pain studies [5,22-24]. A previous double blind 18 week study in painful DPN subjects proved how great the risk for decreased separation of an investigational drug effect from placebo can be [6].That study had in the design a relatively long titration (6 weeks) and, in fact, was considered among the longest trials conducted in patients with painful DPN [6]. Despite most of the inclusion and exclusion criteria are comparable amongst phase 3 investigational programs, there is also some available evidence, that placebo response could reach a plateau in painful DPN studies, but with a significantly shorter duration of the trial design ( 8 weeks) [7]. This debate today still remains open until further research and evaluation is provided.

From the Proof of Concept phase, any attempt to mitigate the placebo response should be welcomed and this approach might allow a new pain medication to successfully progress towards later stages of clinical development.

Compared to the past, randomised, double blind, placebo controlled studies are now essential to establish efficacy for new drugs in neuropathic pain [25].

Typically, drugs approved for neuropathic pain indications lose their therapeutic response over time and have far from an ideal tolerability risk profile. Therefore, where there is an established and available treatment option, a three-arm study (study drug-comparatorplacebo) should be considered to allow the assessment of comparative efficacy and safety of a new product [25].

If positive results in phase 3 are confirmatory of those produced from a PoC study, ultimately this could lead to the approval of a new drug for treating painful DPN. As of today this remains a challenge in a therapeutic area of a well-recognized unmet medical need.

The dangers due to the scale of use and the often abuse or disuse of opioids, anti-depressants, Non-Steroidal Anti-Inflammatory Drugs (NSAIDs) or acetaminophen for treating acute and chronic pain conditions are considerable.

Firstly, a deeper understanding of the pathogenetic mechanisms of neuropathic pain could help to identify responders [26].

Further research is needed to characterise the different patient's phenotypes: this would identify those subjects who really need one and/or another already approved pain medication from those who potentially don ' $t$.

Secondly, by mitigating the placebo response in neuropathic pain 
Citation: Merante D, Skouteris G, Malik R (2013) Developing New Molecules for the Treatment of Painful Diabetic Peripheral Neuropathy: Is it Feasible given the Magnitude of the Placebo Response in Proof of Concept Clinical Studies? J Diabetes Metab 4: 305. doi:10.4172/21556156.1000305

Page 4 of 4

clinical trials it may significantly expedite the development of new analgesic treatments for the benefit of public health [27]. Nowadays it is certainly more challenging than in the past to develop a new treatment for painful DPN because of the change of the patients clinical profiles particularly over the last decade due to the existing therapies [28].

There is a need for gathering data from comparative studies. Also today's patients are more difficult to treat than in the past and combination therapies are often required as multiple pain treatment failures occur over time [2].

Combination therapies include drugs like opioids and morphine in addition or replacement to others, such as tricyclic anti-depressants, selective serotonin reuptake inhibitors, serotonin and noradrenalin reuptake inhibitors, alpha delta 2 ligands. Nevertheless, paracetamol/ acetominophen and aspirin or NSAIDs are also commonly utilized in clinical practice and in clinical research. In clinical setting they are defined as 'rescue medications', even though they are more involved in treating inflammatory rather than neuropathic pain.

As patients with painful DPN suffer from diabetes and many related long-term complications, investigating a new treatment for painful DPN becomes far more challenging than optimization of glucose control. It is of course important, whilst testing a new pain medication for painful DPN, to maintain stable glucose control for the whole duration of the study.

We witness how challenging it is to manage patients' expectations and their hope to feel better during their painful DPN condition. Patients suffering from this complication expect to see an improvement of quality and quantity of sleep. They would welcome any improvement of their pain in the hope to reduce its interference on their daily activities.

A more efficient discovery process leading to the development of better than existing treatments for painful DPN could be obtained by reducing the placebo response in clinical studies.

At the same time any attempt to develop more effective and better tolerated drugs, this could represent a significant step forward for a significant improvement in the quality of life of patients suffering from painful DPN.

\section{References}

1. Tesfaye S, Kempler P (2005) Painful diabetic neuropathy. Diabetologia 48: 805807.

2. Tesfaye S, Boulton AJ, Dickenson AH (2013) Mechanisms and management of diabetic painful distal symmetrical polyneuropathy. Diabetes Care 36: 24562465 .

3. Häuser W, Bartram-Wunn E, Bartram C, Reinecke H, Tölle T (2011) Systematic review: Placebo response in drug trials of fibromyalgia syndrome and painfu peripheral diabetic neuropathy-magnitude and patient-related predictors'. Pain 152: $1709-1717$

4. Hewitt DJ, Hargreaves RJ, Curtis SP, Michelson D (2009) Challenges in analgesic drug development. Clin Pharmacol Ther 86: 447-450.

5. Katz J, Finnerup NB, Dworkin RH (2008) Clinical trial outcome in neuropathic pain: relationship to study characteristics. Neurology 70: 263-272.

6. Ziegler D, Hidvégi T, Gurieva I, Bongardt S, Freynhagen R, et al. (2010) Efficacy and safety of lacosamide in painful diabetic neuropathy. Diabetes Care 33: 839-841.

7. Rosenstock J, Tuchman M, LaMoreaux L, Sharma U (2004) Pregabalin for the treatment of painful diabetic peripheral neuropathy: a double-blind, placebocontrolled trial. Pain 110: 628-638.

8. Segerdahl M (2009) Improving early clinical drug development for neuropathic pain by improving patient selection. Pain 141: 4-5.
9. Dworkin $\mathrm{RH}$, Katz J, Gitlin MJ (2005) Placebo response in clinical trials of depression and its implications for research on chronic neuropathic pain Neurology 65: S7-19.

10. Freeman R, Durso-Decruz E, Emir B (2008) Efficacy, Safety, and Tolerability of Pregabalin Treatment for Painful Diabetic Peripheral Neuropathy. Findings from seven randomized, controlled trials across a range of doses. Diabetes Care 31: 1448-1454

11. Arnold LM, Clauw DJ, Wohlreich MM, Wang F, Ahl J, et al. (2009) Efficacy of duloxetine in patients with fibromyalgia: pooled analysis of 4 placebo-controlled clinical trials. Prim Care Companion J Clin Psychiatry 11: 237-244.

12. Walsh BT, Seidman SN, Sysko R, Gould M (2002) Placebo response in studies of major depression: variable, substantial, and growing. JAMA 287: 1840-1847.

13. Schatzberg AF, Kraemer HC (2000) Use of placebo control groups in evaluating efficacy of treatment of unipolar major depression. Biol Psychiatry 47: 736-744.

14. Fava M, Evins AE, Dorer DJ, Schoenfeld DA (2003) The problem of the placebo response in clinical trials for psychiatric disorders: culprits, possible remedies and a novel study design approach. Psychother Psychosom 72: 115-127.

15. Zimbroff DL (2001) Patient and rater education of expectations in clinical trials (PREECT). J Clin Psychopharmacol 21: 251-252.

16. Giesecke T, Williams DA, Harris RE, Cupps TR, Tian X, et al. (2003) Subgrouping of fibromyalgia patients on the basis of pressure-pain thresholds and psychological factors. Arthritis Rheum 48: 2916-2922.

17. Spallone V, Greco C (2013) Painful and painless diabetic neuropathy: one disease or two? Curr Diab Rep 13: 533-549.

18. Greist JH, Mundt JC, Kobak K (2002) Factors contributing to failed trials of new agents: can technology prevent some problems? J Clin Psychiatry 63: 8-13.

19. Yang $\mathrm{H}$, Cusin $\mathrm{C}$, Fava $\mathrm{M}(2005)$ Is there a placebo problem in antidepressant trials? Curr Top Med Chem 5: 1077-1086.

20. Hearn L, Derry S, Moore RA (2012) Lacosamide for neuropathic pain and fibromyalgia in adults. Cochrane Database Syst Rev 2: CD009318.

21. Selvarajah D, Gandhi R, Emery CJ, Tesfaye S (2010) Randomized placebocontrolled double-blind clinical trial of cannabis-based medicinal product (Sativex) in painful diabetic neuropathy: depression is a major confounding factor. Diabetes Care 33: 128-130.

22. Thienel U, Neto W, Schwabe SK, Vijapurkar U; Topiramate Diabetic Neuropathic Pain Study Group (2004) Topiramate Diabetic Neuropathic Pain Study Group. 'Topiramate in painful diabetic polyneuropathy: findings from three double-blind placebo-controlled trials'. Acta Neurol Scand 110: 221-231.

23. Kingery WS (1997) A critical review of controlled clinical trials for peripheral neuropathic pain and complex regional pain syndromes. Pain 73: 123-139.

24. Quessy SN, Rowbotham MC (2008) Placebo response in neuropathic pain trials. Pain 138: 479-483.

25. European Medicines Agency (EMEA) (2007) Guidelines on clinical medicinal products intended for the treatment of neuropathic pain: 1-10.

26. Merante D (2013) Challenges in Painful Diabetic Peripheral Neuropath clinical research. Oral presentation at the $4^{\text {th }}$ World Congress on Diabetes $\&$ Metabolism, Chicago.

27. (2013) A Randomized, Double-Blind, Placebo and Active Comparator Controlled Study of DS-5565 for Treatment of Neuropathic Pain Associated With Diabetic Peripheral Neuropathy. ClinTrial.Gov: http://clinicaltrials.gov/ show/NCT01496365: accessed on October 25, 2013.

28. Freeman R (2013) New and developing drugs for the treatment of neuropathic pain in diabetes. Curr Diab Rep 13: 500-508. 